\title{
A Survey of Reduction the Interference on Cellular Communication System
}

\author{
Tasher Ali Sheikh \\ Computer Science and \\ Engineering \\ National Institute of \\ Technology, \\ Arunachal Pradesh, India- \\ 791112
}

\author{
Deboraj Muchahary \\ Computer Science and \\ Engineering \\ National Institute of \\ Technology, \\ Arunachal Pradesh, India- \\ 791112
}

\author{
Khanjan Changmai \\ Baruah \\ Computer Science and \\ Engineering \\ National Institute of Technology, \\ Arunachal Pradesh, India-791112
}

\begin{abstract}
Interference reduction is the challenging issue in the field of cellular communication system. Due to limited frequency bandwidth and high cost, we have to reuse same frequency band in the different geographic area resulting the CCI and ACI. In this paper we study two different type of interference. In this Survey we also presented the various interference reduction methods and also discuss the reduction process of Signal to interference ratio. Using these two tools we also evaluate the performance of the Signal to interference ratio.
\end{abstract}

\section{Keywords}

Interference, CCI, ACI, IRT, ET, S/I ratio.

\section{INTRODUCTION}

Interference is a major limiting factor in the performance of cellular radio System. It [1], [2] limits capacity and increase number of called dropped. As we know that our existing base station in cellular communication system are generally Omnidirectional, due to this the Signal power radiated throughout the cell area results in increase of the interference and reduce SNR due to undesired users. Although sector antenna will increase capacity of system by dividing entire cell into sector but have the same problem of interface. Emerging trends of mobile communication and ever growing demand of mobile users with[7] high speed data requirements and migrating towards 4G, Co-Channel interference and multipath in urban/dense urban environment will limit the capacity of the network.

In mobile communication there are several sources of interference.viz.an another mobile within the same cell, an under progress call in a neighboring cell, other BS operating in the same frequency band. Interference is more severe in the Urban Areas [4] due to greater RF noise and more number of MS and BS. Interference causes some major problems in mobile communication. As an example of this we can visualize its effect in voice channel and control channel. Interference on Voice Channel causes:

-crosstalk

-Noise in the background.

While Interference on Control Channel causes:

-missed calls.

-Blocked calls.

-Dropped calls.

There are two major types of interference

1. Co-channel interference, $(\mathrm{CCI})$

2. Adjacent channel interference, (ACI)

\section{CO-OCHENNEL INTERFERNCE}

CCI in a cell occurs due to the other cells that uses same frequency set of the cell. These cells [1] using the same frequency set are called co-channel cell.

Resolved Process of CCI

The techniques to resolve the CCI

i. Interference Reconstruction Technique (IRT): Interference reconstruction [4] techniques is based on the reconstructing all users' signal in order to estimated their crosscorelation components in the received signals.

ii. Equalization Techniques (ET): Equalization techniques Consider the multipath access interference as a special (serve) multipath interference case.

iii. Design a Directional antenna: The co-channel interference can be decrease by using directional antenna. This means that each cell is divided into three or six sectors and uses three or six directional antenna at a base station. Each sector is assigning a set of frequency or channel and hence interference between two cells is decrease.

iv. Parasitic Elements: By using parasitic elements we can reduce the CCI.Parasitic antenna can be design as-

$$
\begin{aligned}
& \text { Normal spacing, } \\
& \text { relatively closed spacing }
\end{aligned}
$$

e. Power control: In these techniques we decrease the Base Tran-receiver power which resulting reduced the CCI.As we know that for a particular radio channel, if the cell size decreases the co-channel reuse distance increases and hence the co-channel interference reduces further.

f. Diversity Receiver: The diversity scheme applied at the receiving end of the antenna [1] is an effective technique for reducing interference because any measures taken at the receiving end to improve signal performance will not cause additional interference.

g. Smart antenna: Smart antenna is one of the most promising technologies that will enable a higher capacity in wireless networks by effectively reducing multipath and co-channel interference. Smart Antenna use adaptive beam forming 
algorithms [7] in a dynamic environment continuously adjusting the weight of antenna arrays for creating a beam to track desired users automatically, and minimize interference from other users by placing nulls in their directions. Smart antennas employ a set of radiating elements arranged in the form of an array. The signals from these elements are combined to form a movable or switchable beam pattern that follows the desired user. In a Smart antenna system the arrays by themselves are not smart, it is the digital signal processing that makes them smart. The process of combining the signals and then $[8,11]$ focusing the radiation in a particular direction is often referred to as digital beam forming.

h. Tilting antenna: Tilting a base station antenna down mechanically or electronically is also a way to reduce CCI. This method reduces the strength of the received signal in the horizontal direction [1]. Taking this concept into an account, if the interfering base stations are design with different tilted angles improvement in CCI is obtain, because the two antennas are operating with different signals strength.

\section{CO-CHANNEL SIGNAL TO INTERFERENCE CALCULATION}

The Signal to interference ratio (S/I) for a [3] mobile is

$$
\begin{gathered}
\frac{S}{I}=\frac{\text { Signalpower }}{\text { Interference from } i^{\text {th }} \text { interfering cell }} \\
\frac{S}{I}=\frac{S}{\sum_{i=1}^{n} I_{i}}
\end{gathered}
$$

The average received power at a distance $d$ is

$$
\begin{gathered}
P_{r}=P_{o}\left(\frac{d}{d_{o}}\right)^{-n} \\
P \propto d
\end{gathered}
$$

Where, $P_{o}$ is the received power at a reference distance $d_{o}$ and $\mathrm{n}$ is the path loss exponent.

\subsection{Average Case Signal to Interference \\ Ratio}

If $D_{i}$ is distance of $i^{t h}$ interferer [2], [3], then the received power is proportional to the $\left(D_{i}\right)^{-n}$.

Thus the S/I for a mobile can be written as

$$
\frac{S}{I}=\frac{R^{-n}}{\sum_{i=1}^{m}\left(D_{m}\right)^{-n}}
$$

For the first tier the interference distance is same for all clusters. So that the $\mathrm{S} / \mathrm{I}$ is given by

$$
\begin{aligned}
& \frac{S}{I}=\frac{(D / R)^{-n}}{m} \\
& \frac{S}{I}=\frac{(\sqrt{3 N})}{m}
\end{aligned}
$$

Where $m=6$ because of equidistance cluster from the MS, so that

$$
\frac{S}{I}=\frac{(\sqrt{3 N})}{6}
$$

This is called average case S/I ratio for seven cell cluster.

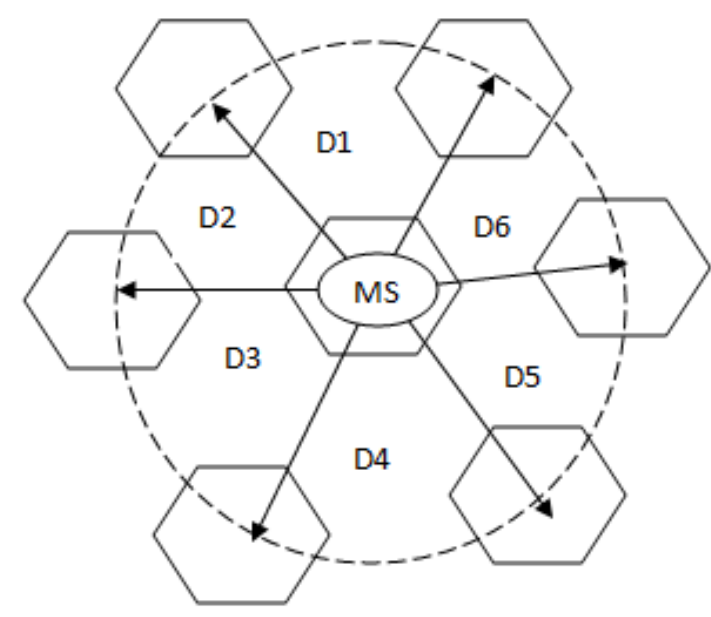

Fig.1: Average Case Signal to Interference Ratio.

\subsection{Worst Case Signal to Interference Ratio}

Assume that MS is in cell boundary. In this case The approximate $\mathrm{S} / \mathrm{I}$ ratio is given by

$$
\frac{S}{I}=\frac{R^{-n}}{2(D-R)^{-n}+2 D^{-n}+2(D+R)^{-n}}
$$

Where,

$\mathrm{R}=$ cell radius,

$\mathrm{D}=$ interference distance, $\mathrm{n}=$ path loss exponent.

$$
\frac{S}{I}=\frac{1}{2(Q-1)^{-n}+2 Q^{-n}+2(Q-1)^{-n}}
$$

Where, $Q=\frac{D}{R}=\sqrt{3 N}$

$\mathrm{N}=$ cluster size

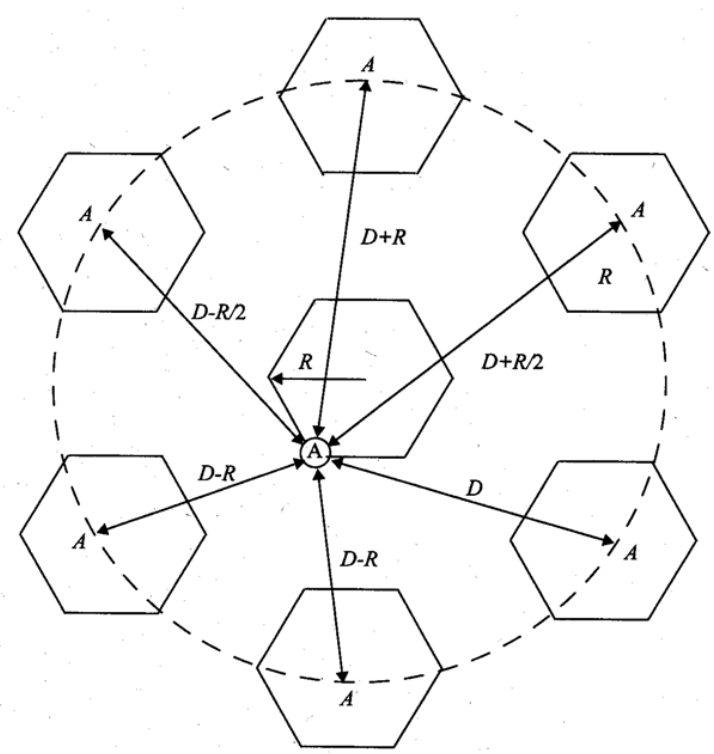

Fig.2: Worst Case Signal to Interference Ratio. 


\section{ADJACENT CHANNEL INTERFERE- NCE (ACI)}

ACI is cause due to the signals [3] that are adjacent in frequency. Adjacent-channel interference (ACI) is basically interference that is created by extraneous power from a signal source in an adjacent channel. Inadequate filtering, such as incomplete filtering of modulation items in frequency modulation (FM) systems, bad tuning, or low quality frequency control, contribute to Adjacent-channel interference.

There are two types of ACI.

I. Imperfect Filtering: Present customers' demand is low cost of handset,i.e less cost of filter which results in more interference. So filtering can't be much more effective at the MS site. The alternate way is to use expensive, very well designed filters at the base station. In fact, [3] the cost of the base station is being shared by so many numbers of users. So, adjacent channel interference is actually handled more at the base stations rather at the handsets level. The problem can be severe if the interferer is very close to the subscriber's receiver. This is because the mobile unit in close proximity has a strong signal which causes adjacent channel interference. Thus resulting in crosstalk at the receiver or if the interference is in control channel, then one of the calls might get dropped.

II. Near Far effect: Another effect of adjacent channel interference is called the near far effect. What is the near far effect? Suppose Transmitter A and Transmitter B are operating on adjacent channels in frequency; when the receiver is far from the desired transmitter and very close to the undesired transmitter, adjacent channel interference is exacerbated. When interference is close to the base station, they radiating in the adjacent channel, while the sub-scriber is actually far away from the base station. It is to note that the path loss exponent is close to four. The signal strength goes down very fast to the power of four of the distance. So if the interfering handset is close to the base station, whereas the subscriber far away from the base station, the signal will get a lot of interference[3] at the base station. Now let us look at it from an example. At first put a base station and the subscriber. So the subscriber is mobile and he is located at a certain distance from the base station. But there are lot of uses and let us have an interfering handset sitting inside a car which happens to be closer to the base station. As bad luck would have it, the interferer is radiating in an adjacent frequency band. So even though the subscriber is trying to communicate with the base station, by the time the signal reaches the base station, it is fairly weak. The path loss exponent [6] is pretty high. So what is being received at the Base station is a low signal level but still it can be handled. It is within the threshold. On the other hand, for the interferer which happens to be located much closer to the base station, it is radiating in the adjacent band and because of the imperfect filters, a lot of energy is leaking in. But it's a lot high energy.

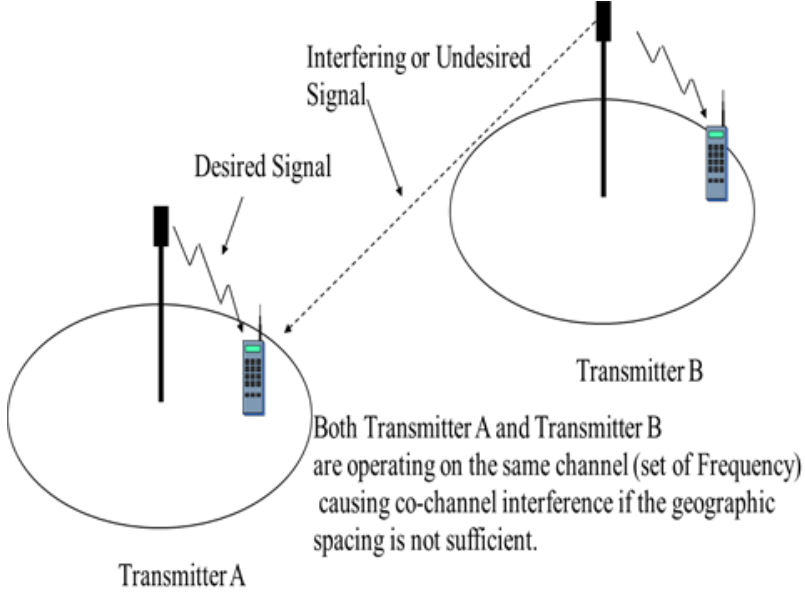

Fig.3: Near Far effect.

\subsection{Resolving Process of ACI}

i. Careful filtering: So the adjacent channel interference can be reduced by careful filtering which means more expensive filters. As shown in Fig.4 our everyday use filter is just like the actual filter, which is causes the interference in the upper channel as well as lower channel as shown in the fig4.Rather than actual filter if we use the "perfect filter" as depicted in Fig.4 there is no interference in the upper channel and lower channel as a result reduces the interference. But the cost of this type of filter is generally so high.

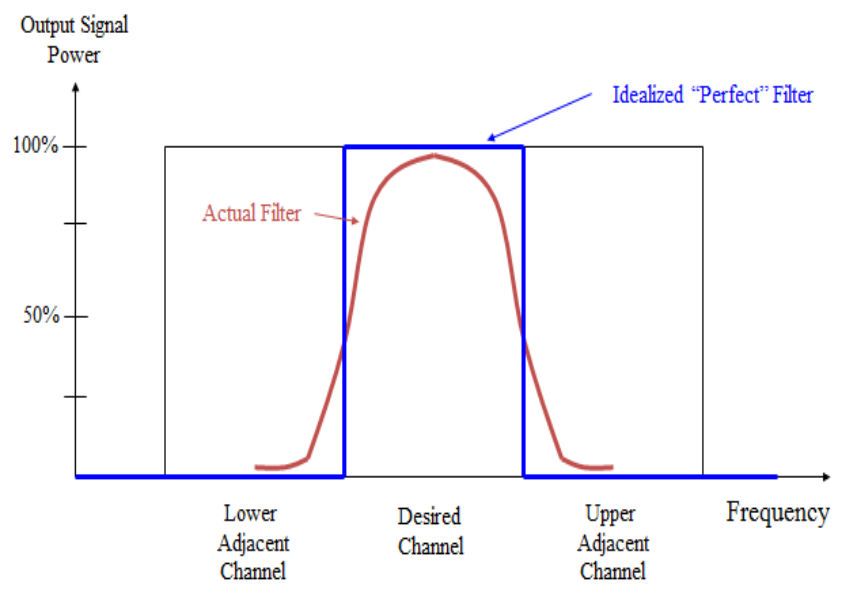

Fig.4: Near Far effect.

ii. Careful channel assignment: The other method to reduce the adjacent channel interference is by smart [2] frequency separation. The frequency band which has to be allocated to different users called channels. Now clearly as and when the demand arises, frequency bands are assigned. But the assignment should not start with the first band and then the consecutive bands. In fact they should have maximum separation. There could be several algorithms for the frequency band allocation. Let's take an example, suppose there are a set of frequency sub bands allocated to a single cell and our job is to allocate these frequency bands[3], [5]. 
But the objective is to ensure that the frequency separation between each channel is maximized. Now it is early in the morning and the first user requests for a channel. So I as a person who is trying to assign different channel gives say, the first channel. It could be any of the channels and I have picked up the first channel. Clearly when second request comes, we should not give the second band or the third band. In fact, I should give the farthest apart. So I'll give the second user the second band which is the farthest apart. Thus the maximum separation will minimize the ACI

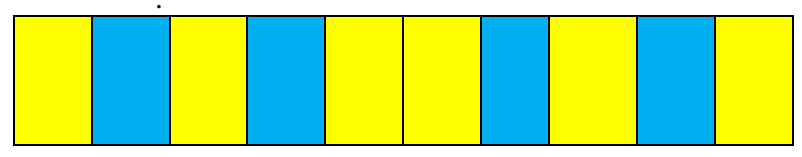

Fig.5: Careful channel assignment

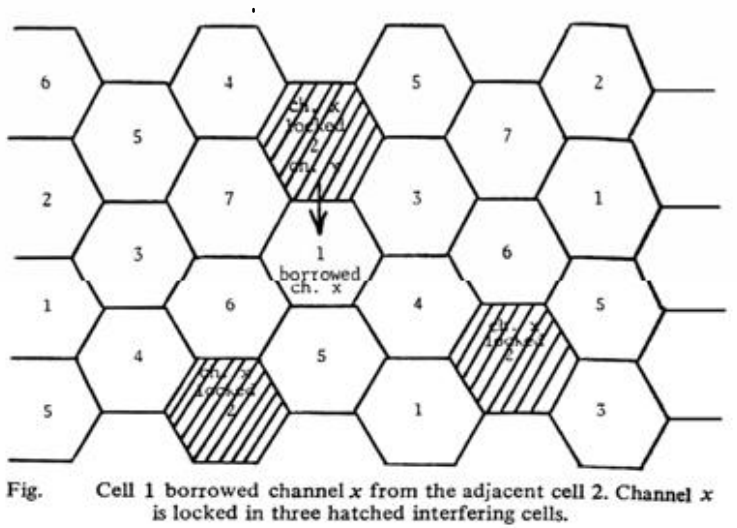

Fig.6: Careful channel assignment

\section{CONCLUSION}

In this paper, we elaborately discuss different kinds of interferences for mobile and wireless communication system namely the co-channel and the adjacent channel interference. We looked at the co-channel interference in greater detail because it is of big concern. We looked at how to calculate the co-channel interference values. Specifically, the average case as well as worst case analysis. We learnt that these two cases have a direct relationship with the path loss exponent being used. Then we looked at the adjacent channel interference. An elaborate $\mathrm{Co}$-Channel Interference (CCI) cancellation technique is evaluated for the mobile and wireless communication system operating in an extreme (worst and average) interference condition. The theoretical analysis and calculation of this system are discussed. Supporting diagram also shows the need of incorporating the error correcting with the CCI cancellation techniques, in order to improve the performance of interference. Also the reliable acquisition of the spreading sequence as well as accurate estimation of the channel parameters must be guaranteed.

\section{ACKNOWLEDGEMENT}

The authors would like to thank Mr. Koj Sombyo, Asst. professor, dept. of CSE, NIT Arunachal Pradesh for his endless support. Also, we would like to thank Prof. (Dr.) C.T.Bhuniya SMIEEE and director of NIT, Arunachal Pradesh, India and S.K.Chakraborty Sir Asst. Prof. of NIT, AP, and India Dept. of CSE for their endless help to complete our work.

\section{REFERENCES}

[1] William C.Y.Lee "Mobile Cellular Telecommunication "2nd edtn Tata McGraw Hill Education Private ltd.

[2] T.S.Rappaport "Wireless communication Principles and Practice" 2nd edtn Pearson publication.

[3] Prof. Dr. Ranjan Bose "Wireless Communication on interference and system capacity L\#6".

[4] Shaheen.K.M, Gupta, Someshwa C. "Computer and Communications ".IEEE-14th International phoenix conference Pub. Year-1995 Pages 678-683.

[5] P. Li†, N. Scalabrino*†§, Y. Fang †, E. Gregori§ and I. Chlamtac* † "Channel Interference in IEEE 802.11b Systems” IEEE GLOBECOM 2007.Page no.887-891.

[6] Kame1 M. Shaheen \& Someshwar C. Gupta "CoChannel Interference Cancellation for DS-SS CDMA Mobile Communication System" 1995 IEEE. Page no.678-683.

[7] Prof.B.G. Hogade, Ms. Sheetal Wadhe , Dr.Shrikant K.Bodhe "Mitigating the Effect of CCI and Multipath in Mobile Communication using Smart Antenna"IJERA ISSN:2248-9622 and National Conference on Emerging Trends in Engineering \& Technology (VNCET-30 Mar'12) Page.no.366-370.

[8] Jack H. Winters. Smart Antennas for Wireless Systems, IEEE Personal Communications, February 1998.

[9] W.C.Y.Lee "Mobile Communication Design Fundamental “, John Wiley \& Son, 1993, PP 30-31.

[10] W.C.Y.Lee "Mobile Communication Design Fundamentals“, John Wiley \& Son, 1993, PP 88-94.

[11] A. F. Eric OH. Smart Antennas and Dynamic Sector Synthesis, undergraduate thesis, University of Queensland, School of Information Technology and Electrical Engineering, 2001. 